\title{
Can We Detect Submesoscale Motions in Drifter Pair Dispersion?
}

\author{
SEBASTIAN ESSINK \\ MIT-WHOI Joint Program in Oceanography, Woods Hole, Massachusetts \\ VERENA HORMANN AND LUCA R. CENTURIONI \\ Scripps Institution of Oceanography, University of California, San Diego, La Jolla, California \\ AMAla MaHADEVAN \\ Woods Hole Oceanographic Institution, Woods Hole, Massachusetts
}

(Manuscript received 2 September 2018, in final form 5 June 2019)

\begin{abstract}
A cluster of 45 drifters deployed in the Bay of Bengal is tracked for a period of four months. Pair dispersion statistics, from observed drifter trajectories and simulated trajectories based on surface geostrophic velocity, are analyzed as a function of drifter separation and time. Pair dispersion suggests nonlocal dynamics at submesoscales of 1-20 km, likely controlled by the energetic mesoscale eddies present during the observations. Second-order velocity structure functions and their Helmholtz decomposition, however, suggest local dispersion and divergent horizontal flow at scales below $20 \mathrm{~km}$. This inconsistency cannot be explained by inertial oscillations alone, as has been reported in recent studies, and is likely related to other nondispersive processes that impact structure functions but do not enter pair dispersion statistics. At scales comparable to the deformation radius $L_{D}$, which is approximately $60 \mathrm{~km}$, we find dynamics in agreement with Richardson's law and observe local dispersion in both pair dispersion statistics and second-order velocity structure functions.
\end{abstract}

\section{Introduction}

A better understanding of the Lagrangian transport of tracers, energy, and enstrophy in the ocean is relevant to a wide range of problems. It helps describe, for example, how pollutants (e.g., Rypina et al. 2013; Poje et al. 2014), freshwater (e.g., Mahadevan et al. 2016), or biological organisms (e.g., Rypina et al. 2014) disperse and how energy and enstrophy cascade across scales. The two-dimensional Lagrangian statistics of the near-surface oceanic flow and its associated transport can be explored using pairs of drifters, which float at the surface while being advected by the flow a few meters below.

In a turbulent ocean, the separation of pairs of drifters can theoretically be predicted when the kinetic energy spectrum is known (LaCasce 2008, 2016) and, furthermore, depends on the separation distance $r$. On scales above about $100 \mathrm{~m}$, the vertical velocity makes a negligible contribution to the kinetic energy, which is

\footnotetext{
Corresponding author: Sebastian Essink, sessink@mit.edu
}

dominated by the horizontal velocity. The horizontal pair dispersion, defined as the mean square separation of pairs of drifters, describes how a group of drifters disperses around its center of mass. If energy spectra are steep, like the $E \sim k^{-3}$ observed in the enstrophycascading range of two-dimensional turbulence (Charney 1971; Nastrom et al. 1986), where the horizontal wavenumber is defined as $k=\left(k_{x}^{2}+k_{y}^{2}\right)^{1 / 2}$, the pair dispersion is dominated by the largest eddy in that range and termed nonlocal (Bennett 1984). If energy spectra are flatter, like the $E \sim k^{-5 / 3}$ observed in the energycascading ranges of two-dimensional and threedimensional turbulence (Charney 1971; Kraichnan 1967), the pair dispersion is dominated by eddies with length scales similar to the pair separation distance and termed local (Bennett 1984; Richardson 1926).

In the presence of two inertial spectral ranges, pairs of drifters transition from one dispersion regime to another as their separation distances grow (LaCasce 2008). As an illustration, consider a two-dimensional, quasigeostrophic model often used to represent mesoscale dynamics in the ocean. At small separations, initially, 
in the enstrophy-cascading range, drifters disperse nonlocally until they reach the scale at which energy is injected (often through baroclinic instability at the first baroclinic deformation radius $L_{D}$, which we estimate as $60 \mathrm{~km}$ for our observations; Chelton et al. 1998). Subsequently, in the energy-cascading range, they disperse locally. Unlike in the atmosphere (Nastrom et al. 1986; Er-El and Peskin 1981), the kinetic energy spectrum in the ocean and its inertial ranges are not well documented. Subdeformation scale dynamics are not thoroughly understood, are often dominated by ageostrophic, divergent motions (Bühler et al. 2014; Callies and Ferrari 2013; D'Asaro et al. 2018), and vary significantly in time and space (Callies et al. 2015).

At submesoscales, considered as length scales of $0.1-20 \mathrm{~km}$ in our observations, a transition from nonlocal to local dispersion is likely to occur: The two-dimensional, quasigeostrophic flow, with energy spectra of $E \sim k^{-3}$ intensifies at surface fronts and breaks down into smaller eddies and filaments, with energy spectra of $E \sim k^{-2}$ or $E \sim k^{-5 / 3}$. In this range, energetic eddies and filaments are reported in observations (Shcherbina et al. 2013; Gon et al. 2018), as well as in numerical simulations (Thomas et al. 2008), which are likely energized by surface frontogenesis (Lapeyre and Klein 2006; Hoskins 1982; Stone 1966) and mixed layer baroclinic instabilities (Boccaletti et al. 2007; Fox-Kemper et al. 2008). Conceptually, in regions where submesoscale flows and fronts are energetic, pair dispersion is expected to be local and then transitions to a nonlocal regime at larger scales in the enstrophy-cascading range of two-dimensional turbulence. Studying the dispersion at submesoscales is complicated, however, by the multitude of processes that reign the same spatiotemporal band. A careful characterization is thus needed to discern them among inertial oscillations, tides, Langmuir turbulence, and the mesoscale circulation.

The Bay of Bengal, the location of this study (Fig. 1), hosts energetic submesoscale dynamics, which are evident in observations (e.g., Ramachandran et al. 2018) and in numerical simulations (e.g., Sarkar et al. 2016). These likely occur at freshwater-dominated density fronts, which are generated by massive seasonal freshwater fluxes, mainly from major rivers in the north, and intense precipitation during the southwest monsoon. The shallow freshwater cap affects the evolution of the sea surface temperature (SST; Jaeger and Mahadevan 2018) and the upper-ocean's heat content (Shroyer et al. 2016; Mahadevan et al. 2016), both of which can alter the air-sea fluxes and, hence, affect the monsoon dynamics. The Air-Sea Interaction Regional Initiative
(ASIRI; Lucas et al. 2014; Wijesekera et al. 2016) aims to understand the upper-ocean dynamics in the Bay of Bengal by extensive high-resolution measurements and modeling to eventually improve the monsoon forecasts.

The primary objectives of this study are to characterize the near-surface dispersion in the Bay of Bengal (specifically the dispersion of pairs of drifters) and to identify the dominant dispersion regime at submesoscales.

We use high-resolution data from a large cluster of drifters deployed as part of ASIRI in the Bay of Bengal (Hormann et al. 2016) to characterize the near-surface dispersion. Close pairs of drifters that report their position with high temporal resolution allow studying the submesoscale range of motions, which is challenging to observe synoptically with shipboard measurements because of the fast evolution of kilometer-scale features within time scales of hours to days. We compare the statistics of separations and velocities of drifter pairs to the theoretical predictions of local and nonlocal dispersion. Trajectories are low-pass filtered to determine the impact of inertial oscillations and small-scale processes on the dispersion statistics. For comparison, we simulate drifter trajectories using the satellite-derived, geostrophic flow field in the same region and contrast statistics from simulated, "AVISO-advected" drifters to the observed drifters. To identify the dispersion by spatially uncorrelated motions, we add a stochastic closure to the AVISO-advected drifters at small scales which is correlated in time.

In what follows, section 2 introduces the drifter dataset collected in the Bay of Bengal, the simulated drifter experiment based on satellite-derived flow fields, and the metrics used to characterize the nearsurface dispersion. Section 3 presents the results for complementary dispersion statistics and compares drifter statistics to theoretical expectations. Section 4 offers a discussion of the results followed by a conclusion in section 5 .

\section{Data and approach}

\section{a. Drifter deployments}

We launched 46 surface drifters (one of which failed after deployment) during an extensive measurement campaign in the Bay of Bengal in September 2015 as part of ASIRI (Wijesekera et al. 2016; Hormann et al. 2016). The drifters were Surface Velocity Program (SVP) drifters (Niiler 2001; Maximenko et al. 2013) that consist of a buoy and a holey-sock drogue at 15-m depth. SVP drifters are part of the Global Drifter Program (Niiler 2001; Maximenko et al. 2013; Centurioni 2018) that aims to map the near-surface 

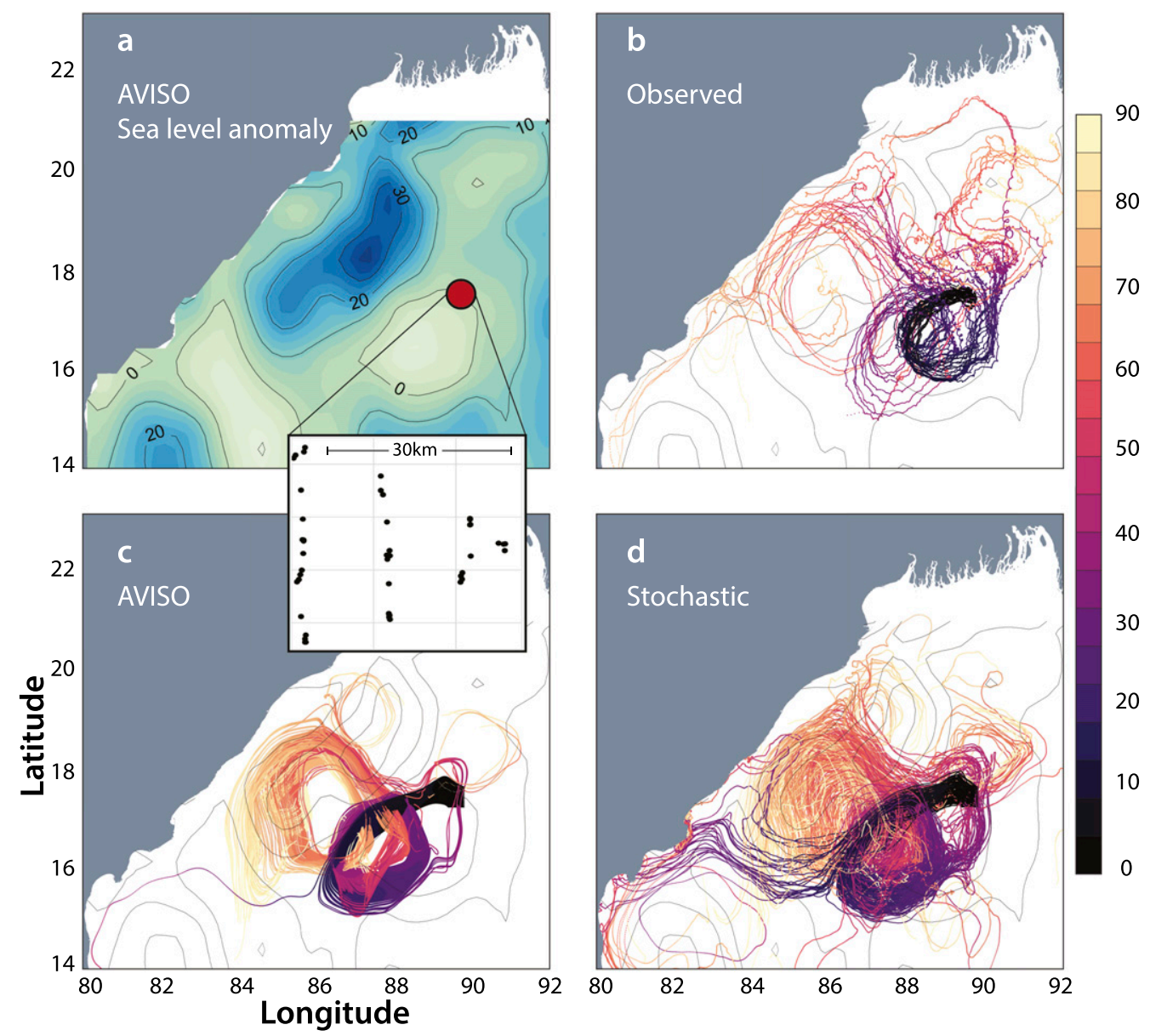

80

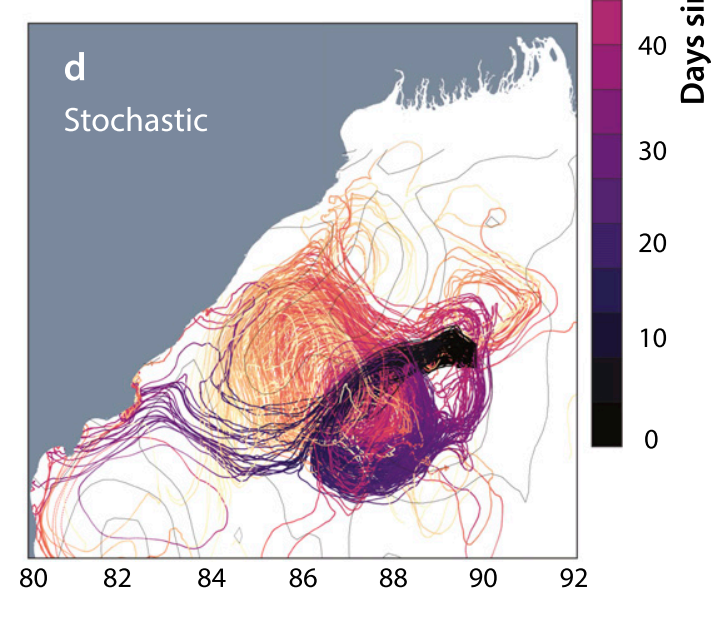

FIG. 1. (a) AVISO-derived sea level anomaly in the northern Bay of Bengal on the day of the drifter release (2 Sep 2015), with the drifter-release location indicated in red and the initial drifter positions overlaid. Trajectories of the (b) observed drifters, (c) AVISO-advected, and (d) stochastic drifters (AVISO-advected with stochastic closure at small scales). Color denotes time after deployment with darker colors indicating early times after deployment.

circulation of the global ocean and to provide SST and sea level pressure data. These data are important for calibration and validation of satellite-derived SST datasets (e.g., Zhang et al. 2009) and for numerical weather prediction (Centurioni et al. 2017; Horányi et al. 2017).

Drifters were released at the edge of a mesoscale cyclonic eddy and across a strong salinity and density front (Figs. 1a,b). With the goal of resolving motions over a wide range of length scales, we deployed drifters such that pair separations ranged from 0.5 to $30 \mathrm{~km}$. We achieved this by deploying 10 clusters of four drifters (Hormann et al. 2016) as shown in the inset in Fig. 1. Each cluster of four drifters was deployed at almost the same time and provides six drifter pairs with a minimum separation of less than $1 \mathrm{~km}$. The entire array of 10 clusters, with an intercluster separation of $5 \mathrm{~km}$, was deployed over a period of $48 \mathrm{~h}$.

During the first month after deployment, drifters reported their positions every $5 \mathrm{~min}$ (Hormann et al. 2016) giving a particularly high temporal resolution. After that, drifters reported every $30 \mathrm{~min}$.

A conservative estimate of the position error is $50 \mathrm{~m}$, which is likely a function of the region, sea state, and GPS coverage. The position data were quality controlled to remove erroneous GPS fixes and median filtered with a 1-h window to remove spurious events of acceleration. The velocity data of each drifter were then calculated by centered differencing. We bin the data to a 30-min grid for the first month and a 1-h grid after that by taking the median value of all points in this period. For the 90 days considered in this study, all drogues stayed attached, thus ensuring that they followed $15-\mathrm{m}$ depth currents with an accuracy of $\sim 0.1 \mathrm{~m} \mathrm{~s}^{-1}$ in winds up to $10 \mathrm{~m} \mathrm{~s}^{-1}$ (Niiler et al. 1995).

Since the drifters have a high temporal resolution, they resolve processes such as near-inertial oscillations and tides, and Langmuir turbulence. We assume that the effect of wind and surface waves on the drifter positions 

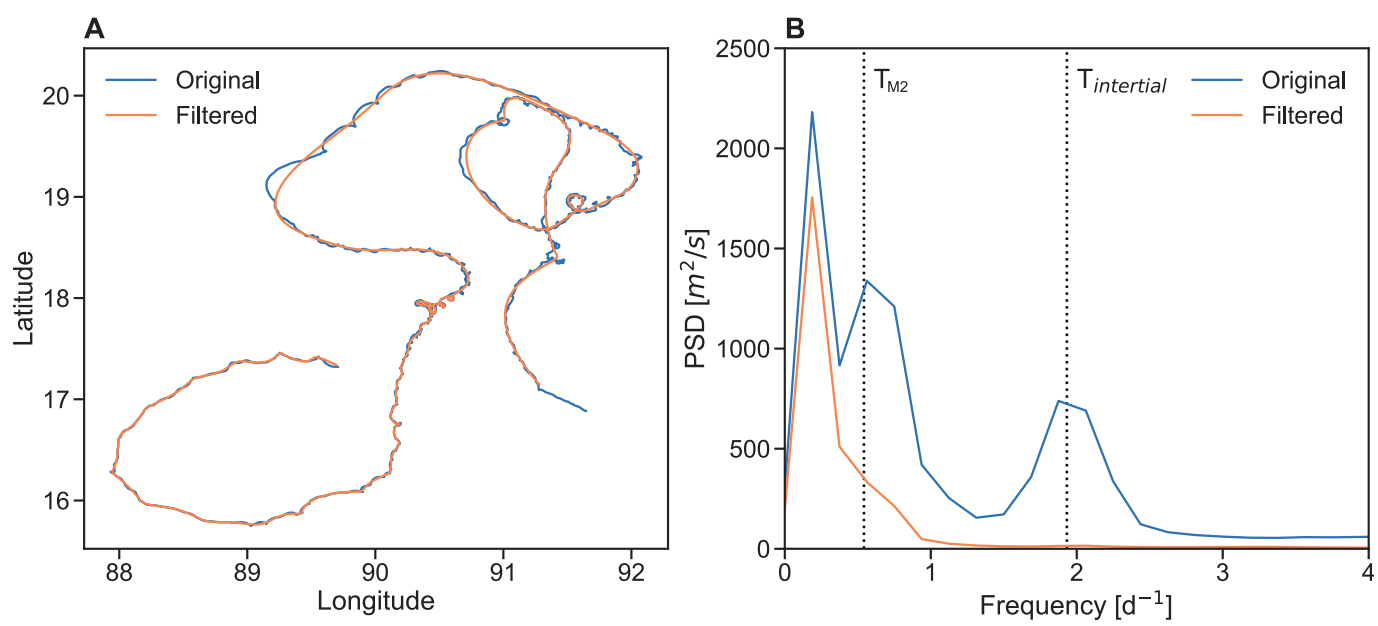

FIG. 2. (a) Example trajectory for one drifter experiencing inertial oscillations. Unfiltered and low-pass-filtered trajectories are indicated in blue and orange, respectively. (b) Ensemble mean velocity power spectral density, before (blue) and after (orange) low-pass filtering with a cutoff of 1.5 times the inertial period. Dotted lines indicate the inertial and $\mathrm{M}_{2}$ tidal frequencies. The inertial and tidal spectral peaks seen in the original data (blue) are removed from the processed data by filtering (orange).

is small since their drogues are at 15-m depth and the Stokes' drift is a second-order effect on the drifter displacement (Niiler et al. 1995). The Lagrangian frequency spectra of the observed drifters show high energy density at the inertial frequency and the $\mathrm{M}_{2}$ tidal frequency (Fig. 2; cf. Hormann et al. 2016), however, substantially less than at subinertial frequencies. For part of the subsequent analysis, we low-pass filter the position data using a fifth-order Butterworth filter with a cutoff of 1.5 times the inertial period $T_{\text {inertial }}$ (Fig. 2). The velocity is then recomputed by finite differencing. Filtering removes both inertial oscillations and motions at the tidal frequencies (Fig. 2). By applying the filter in forward and backward direction, edge effects are minimized. The low-pass-filtered dataset allows us to separate the effect of near-inertial motions on the dispersion statistics.

\section{b. AVISO-advected drifters}

As a reference dataset for the large-scale, geostrophic circulation, we simulate drifters that are advected by satellite-derived, geostrophic currents $\mathbf{U}_{\text {geo }}$ computed from the daily, delayed-time AVISO sea level anomaly (Fig. 1c; Le Traon et al. 1998; Ducet et al. 2000). The sea level anomaly product is gridded to $1 / 4^{\circ}$, which is about half the local deformation radius $L_{D}$.

We trace the trajectories of the AVISO-advected drifters by time-integrating the currents using a fourthorder Runge-Kutta scheme and an hourly time step (Fig. 1c). This time step is well below the temporal resolution of AVISO; the geostrophic velocities $\mathbf{U}_{\text {geo }}$ are therefore interpolated linearly in time. Cubic spatial interpolation is used to find the velocity at each drifter position.

To compare with the observed trajectories, we release the AVISO-advected drifters in the same region in the Bay of Bengal and at the same time as the real drifters. Since we are interested in pairs of drifters, we initialize pairs of drifters with fixed initial separation creating a grid of $N$ drifters and adding a second grid offset by a distance $\mathbf{r}_{0}$, where separation $\mathbf{r}_{0}$ is the minimum distance that a pair of drifters is separated. We simulate 200 AVISO-advected drifters, which provide a combination of 19900 drifter pairs at every time step, at least 100 of which have the minimum initial separation.

\section{c. Stochastic drifters}

Lagrangian trajectories of fluid parcels are significantly altered by small-scale processes that influence the dispersion properties, for example, how fast they spread from their source region or for how long they are trapped in a flow feature.

First-order stochastic models give reasonably realistic results when modeling drifter trajectories in the ocean (Griffa et al. 1995; LaCasce 2010; Koszalka et al. 2009; Haza et al. 2012). As opposed to zeroth-order models, which are stochastic in the drifter positions, first-order models are stochastic in the velocity. The first-order stochastic model velocities $u_{i}$ include an exponentially fading memory of the past velocity [first term on the right-hand side in Eq. (1)] and a noise increment $d \omega$ of a continuous Wiener process $W(t)$ [second term on the right-hand side in Eq. (1)]. The new drifter position $x_{i}$ along a trajectory is then found by integrating the 
following stochastic differential equation for the $x$ component (and analogously for the $y$ component) of the position:

$$
\begin{aligned}
& d u_{i}=-\frac{1}{T_{L}} u_{i} d t+\sqrt{\frac{2}{T_{L}}} \nu d \omega, \\
& d x_{i}=\left(u_{i}+u_{\mathrm{geo}}\right) d t,
\end{aligned}
$$

where $U_{\text {geo }}$ is the background, AVISO-derived current, $\nu=\left\langle u_{i}^{2}\right\rangle^{1 / 2}$ the mean square single particle velocity, which can be interpreted as the turbulent velocity fluctuations, and $T_{L}$ the $e$-folding time of the memory loss.

We take $T_{L}$ to be the Lagrangian time scale estimated at 1 day from the velocity autocorrelation of the highpass-filtered drifter trajectories [with cutoff frequency (2/3) $T_{\text {inertial }}$. As Koszalka et al. (2009) suggest, we then tune the amplitude of the noise such that the relative dispersion matches the observed relative dispersion. We arrive at a value of $\nu=0.02 \mathrm{~m} \mathrm{~s}^{-1}$ for the turbulent velocity fluctuation, which is similar to the value found by Haza et al. (2012).

We use the first-order stochastic model to account for the subgrid processes and superpose it onto the AVISO-derived background currents $\mathbf{U}_{\text {geo }}$ (Fig. 1d). The resulting stochastic trajectories deviate significantly from deterministic AVISO-advected trajectories due to small-scale perturbations that displace drifters enough to leave geostrophic streamlines. The AVISO-advected drifters lack this small-scale variability. Furthermore, the added stochastic component $\mathbf{u}_{i}$ to the mean flow is uncorrelated in space.

In the following sections, we will refer to three different datasets as 1) the observed drifters, 2) the AVISOadvected drifters, and 3 ) the stochastic drifters, which are the AVISO-advected drifters with stochastic noise.

\section{d. Dispersion metrics and structure functions}

\section{1) RELATIVE DISPERSION}

A common metric for the dispersion of two particles is the mean square separation, known as relative dispersion $D^{2}$ (e.g., Richardson and Stommel 1948; LaCasce 2008; Lumpkin and Elipot 2010), where the separation is the magnitude of the separation vector $r=|\mathbf{r}|$. Relative dispersion is the ensemble average of $r^{2}$ over all pairs that are closer than a small distance $\delta$ at some time during the drift.

Here we use original ( $r<\delta$ initially) and chance pairs $(r<\delta$ at a later time during the drift; LaCasce 2008). Thanks to the dense initial deployment of drifters, the majority of drifter pairs are original pairs (about 92\%).
Relative dispersion is then defined as the variance of pair separation distances of the selected $N$ pairs:

$$
D^{2}(t)=\frac{1}{N} \sum_{i \neq j}^{N}\left\|\mathbf{x}_{i}(t)-\mathbf{x}_{j}(t)\right\|^{2} .
$$

Trajectories are sorted such that they begin with the time of minimum separation. The time is thus relative to the time of minimum separation $t=t-t_{0}$. The relative diffusivity is defined as the rate of change of the relative dispersion

$$
\kappa_{\mathrm{rel}}(t)=\frac{1}{2} \frac{d}{d t} D^{2}(t)
$$

and is often binned as a function of separation $r$.

Relative dispersion $D^{2}$ strongly depends on the correlation between velocities of pairs of drifters (LaCasce and Ohlmann 2003; Koszalka et al. 2009). The earlytime and long-time limits are trivial. At early times, when pair separations are small, pair velocities differ approximately by a linear shear. The pair velocity correlation is thus constant, and pairs spread like $t^{2}$. At large separations, when pair separations reach the scale of the energy-containing eddies, pair velocities become uncorrelated, and the relative dispersion resembles a random walk with constant particle diffusivity. This diffusivity asymptotes to twice the diffusivity with which a single particle spreads around its initial location. The ensemble-average single particle diffusivity is also called absolute diffusivity (LaCasce and Bower 2000; Kirwan et al. 1978).

At intermediate scales, however, the relative dispersion can be predicted from turbulence scaling laws given the energy spectrum, $E \sim k^{-\alpha}$, and is often referred to as turbulent dispersion. It can then be shown that in a nonlocal dispersion regime with $\alpha \geq 3$, here referred to as the Lundgren regime, we expect the relative dispersion to grow exponentially in time (Lundgren 1981; Lin 1972). In a local regime with $1<\alpha<3$, we expect relative dispersion to grow as a power law $D^{2} \sim t^{4 /(3-\alpha)}$ (Foussard et al. 2017). If $\alpha=5 / 3$, relative dispersion grows like $D^{2} \sim t^{3}$, subsequently termed Richardson dispersion (Richardson 1926; Morel and Larceveque 1974). Similarly, we expect scaling laws to hold for the relative diffusivity. For a self-similar energy spectrum of the form $E \sim k^{-\alpha}$, it can be shown that $\kappa_{\text {rel }} \sim r^{(\alpha+1) / 2}$ (Bennett 1984; LaCasce 2008). This scaling law reproduces $\kappa_{\text {rel }} \sim r^{4 / 3}$, Richardson 4/3 law (Richardson 1926), for $\alpha=5 / 3$, and $\kappa_{\text {rel }} \sim r^{2}$ for $\alpha=3$.

We estimate the $95 \%$ confidence interval of the relative dispersion and diffusivity by bootstrapping. For each time, the population of available pairs is sampled 1000 times with replacement and the statistics are 
computed on each subsample. The confidence interval is then determined from the distribution of values that the subsamples generate.

\section{2) Finite-SIZE LyAPUNOV EXPONENT}

Since pair dispersion is not only a function of the velocity field, but also of the pair separation itself, it is necessary to adopt metrics that invoke distance as their independent variable. Distance-averaged statistics treat the positions and velocities as Eulerian point measurements on an unstructured grid. Although Eulerian and Lagrangian statistics should be equivalent, sparse drifter trajectories often yield different results between timeaveraged and distance-averaged metrics.

The Lyapunov exponent is a measure of the rate of divergence of neighboring trajectories. It is widely used to identify trajectories that separate exponentially fast (Artale et al. 1997; Aurell et al. 1997) and is a technique borrowed from the dynamical systems approaches to study coherent structures in the flow (Haller 2015). To compute the finite-size Lyapunov exponent (FSLE; e.g., LaCasce and Ohlmann 2003; LaCasce 2008), we create an array of distance classes $r_{n}=r_{0} \varepsilon^{n}, n=1,2,3, \ldots$, where $\varepsilon=\sqrt{2}$ is an arbitrary constant, and record the time $T_{n}$ that each pair takes to separate by a finite distance $r_{n}$. The FSLE $\lambda_{n}$ are then determined by

$$
\lambda_{n}=\log (\varepsilon) \frac{1}{\left\langle T_{n}\right\rangle},
$$

where $\langle\cdot\rangle$ is the ensemble average over all pairs. Contrary to the relative dispersion, all drifter pairs regardless of their initial separation are taken into account.

In theory, Lyapunov exponents are used to study exponential growth of pair separations. If the FSLE is constant over a range of distance classes, the $e$-folding time scale is constant, which is equivalent to an exponential growth of pair separations. However, FSLEs are also useful to study growth of separations that is not exponential; we can use scaling arguments to relate the FSLE power law exponent $\beta$ to the mean square separation $D^{2}$ as $D^{2} \sim t^{-2 / \beta}$.

Previous studies have shown that FSLEs are sensitive to the temporal evolution of the data (LaCasce and Ohlmann 2003; Poje et al. 2010), as well as the implementation of the method (Lumpkin and Elipot 2010; Haza et al. 2008). If the temporal resolution is not high enough, FSLEs underestimate the maximum Lyapunov exponent and miss transitions between regimes. Similarly, the quality of drifter data affects the estimate, as small-scale noise in the position data contaminates the FSLE at scales that are up to 6 times larger than the noise scale (Haza et al. 2014).
Here, we use the method of fastest crossing (e.g., Lumpkin and Elipot 2010; Haza et al. 2008) to determine $T_{n}$. While the method of first crossings (e.g., LaCasce 2008) only accounts for the first time a pair's separation grows from one distance class to the next, we break up the time series of pair separation into monotonically increasing segments and record every incident that a pair crosses a distance class. By counting every crossing, we increase the number of data points per distance class and alleviate the problem of small numbers of pairs. Furthermore, our half-hourly pair separations never exactly coincide with the edges of distance bins. As suggested by Haza et al. (2008), we linearly interpolate the time it would take to exactly reach the bin edge, thus, increasing the accuracy of crossing times and compensating for the limited temporal resolution of the data.

We estimate the $95 \%$ confidence interval of the FSLE by bootstrapping. For each separation bin, we generate 1000 subsets of the available crossing times by randomly resampling the data with replacement. For each subset, the FSLE is computed with the method described above. The confidence interval is then determined from the distribution of values that the subset generate.

\section{3) PAIR SEPARATION PDF}

Richardson (1926) was the first to distinguish dispersion regimes by studying pair separation probability density functions (PDFs) $p(r, t)$ in what he called "distance-neighbor functions." These describe the probability that a pair released with initial separation $r_{0}$ will have separation $r$ at time $t$. PDFs and their statistics are powerful because they illuminate the pair dispersion process (Sullivan 1971) and encapsulate conventional pair dispersion statistics; the second moment of the pair separation PDF, for instance, is the relative dispersion. By comparing PDFs to the theoretically expected solutions for a given flow field, we can distinguish between different turbulent dispersion regimes (LaCasce 2010; Beron-Vera and LaCasce 2016; Bennett 1984; Graff et al. 2015).

In theory, if the energy spectrum $E(k)$ is known, we can derive a scaling prediction for the diffusion coefficient $\kappa_{2}$. Using the initial condition $p(r, t=0)$, we can then describe the evolution of the pair separation PDFs with a Fokker-Planck equation (e.g., BeronVera and LaCasce 2016; Bennett 2006):

$$
\frac{\partial p}{\partial t}=\frac{1}{r} \frac{\partial}{\partial r}\left(r \kappa_{2} \frac{\partial p}{\partial r}\right)
$$

For uncorrelated, normally distributed drifter velocities, the diffusion parameter $\kappa_{2}$ is constant and equal to twice 
the single-particle diffusivity. In this case, the pair separation PDF resembles a Rayleigh distribution (Beron-Vera and LaCasce 2016). In turbulent flow, however, drifter velocities are correlated and we can find solutions for the Fokker-Planck equation as a function of time and separation. For a given energy spectrum, for example, the energy spectrum associated with the Richardson regime (Richardson 1926) regime or the Lundgren regime (Lundgren 1981), the diffusivity $\kappa_{2}$ can be determined. Good summaries and derivations can be found in LaCasce (2010) and Bennett (2006).

To compare our data to the theoretical PDFs of the Lundgren, Richardson, and Rayleigh regimes, we first estimate $\kappa_{2}$ for each of the regimes. In the Richardson regime, the relative diffusivity is $\kappa_{2}=\beta r^{4 / 3}$, where $\beta$ is related to the third root of the energy dissipation rate (Graff et al. 2015; LaCasce 2010; Beron-Vera and LaCasce 2016). We find $\beta$ by fitting the theoretical prediction for the relative dispersion to the data. These fits are only computed over the initial period until separations are 10 times as large as the initial separation $r_{0}$. Analogously, in the Lundgren regime, the relative diffusivity is $\kappa_{2}=r^{2} / T$, where $T$ is related to the third root of the enstrophy dissipation rate (Graff et al. 2015; Beron-Vera and LaCasce 2016; LaCasce 2010). Parameter $T$ is found similarly, by fitting the theoretical prediction of relative dispersion to our data for scales $r<10 r_{0}$. For the Rayleigh regime, the diffusion parameter is $\kappa_{2}=\left\langle r^{2}\right\rangle / 2 t$, which is twice the single particle diffusivity. We determine $\kappa_{2}$ from the mean of the relative dispersion for $t>20$ days.

Using $\kappa_{2}$, theoretical predictions can be made for the pair separation PDFs and their moments. The PDF for Richardson dispersion is self-similar with a kurtosis that asymptotes to 5.6 in the long-time limit. The PDF for nonlocal dispersion is lognormal and becomes more peaked with time; its kurtosis grows exponentially with time. The PDF for uncorrelated velocities has a kurtosis of 2 for a Rayleigh distribution (Bennett 1987; Beron-Vera and LaCasce 2016).

\section{4) STRuCture FUnCTIONS}

The variance of the pair velocity differences $\delta u=\left\langle\left\|\mathbf{u}_{i}-\mathbf{u}_{j}\right\|\right\rangle$ is defined as the second-order velocity structure function $S_{2}=\left\langle\delta u^{2}\right\rangle$. Separating the velocity $\mathbf{u}$ into its longitudinal, $\mathbf{u}_{l}=[(r \cdot \mathbf{u}) /|r|] \hat{\mathbf{l}}$, and transverse, $\mathbf{u}_{t}=\{[(r \times \mathbf{u}) \cdot \hat{\mathbf{k}}] /|r|\} \hat{\mathbf{t}}$, components (where $\hat{\mathbf{l}}$ and $\hat{\mathbf{t}}$ are unit vectors in the longitudinal and transverse direction, respectively) (Bühler et al. 2014; Balwada et al. 2016; Babiano et al. 1990), we define the longitudinal $\left(S_{l}\right)$ and transverse $\left(S_{t}\right)$ structure functions as

$$
\begin{aligned}
& S_{l}(r)=\left\langle\left\|\mathbf{u}_{l_{i}}-\mathbf{u}_{l_{j}}\right\|^{2}\right\rangle, \\
& S_{t}(r)=\left\langle\left\|\mathbf{u}_{t_{i}}-\mathbf{u}_{t_{j}}\right\|^{2}\right\rangle,
\end{aligned}
$$

where $\langle\cdot\rangle$ denotes the average over separation distances of all distinct drifter combinations $i, j$, and $r$ is the magnitude of the separation vector. Here, we take the average of the squared velocity difference for the computation of the structure function.

In theory, the second-order velocity structure function is related to the energy spectrum by a Hankel-Fourier transform (Bennett 1984; LaCasce 2016),

$$
S_{2}(r)=2 \int_{0}^{\infty} E(k)\left[1-J_{0}(k r) d k\right],
$$

where $J_{0}$ is the zeroth-order Bessel function.

Due to the difficulty of observing the energy spectrum in the ocean, structure functions have been used to understand the distribution of energy across scales. As LaCasce (2016) points out, however, the calculation of energy spectra from drifter-derived structure functions is not practicable. Particularly at large scales, where a limited number of pairs is available and only a few realizations of the flow are sampled, the transformation to energy density produces large uncertainties. Nonetheless, structure functions give valuable information about the distribution of energy as a function of scale (Balwada et al. 2016). In particular, they are able to reproduce the scale-dependent transitions from one inertial subrange to another in the energy spectrum.

Examining the asymptotic limits of the integral in Eq. (9) offers illuminating physical insights (Bennett 1984; Balwada et al. 2016; Babiano et al. 1990):

$$
S_{2}(r)=\frac{r^{2}}{2} \int_{0}^{2 / r} k^{2} E(k) d k+2 \int_{2 / r}^{\infty} E(k) d k
$$

where $r$ is the pair separation distance, $E(k)$ is the kinetic energy spectrum, and $k$ is the horizontal wavenumber. In the limit of $r \rightarrow 0$, structure functions are dominated by the enstrophy provided by velocity gradients of the largest eddies in the flow. In the limit of $r \rightarrow \infty$, structure functions are dominated by the energy of eddies of the same scale as $r$.

We can predict the power law behavior of structure functions from Eq. (10). Given a self-similar energy spectrum $E(k) \sim k^{-\alpha}$, the structure function scale like $S_{2} \sim r^{\alpha-1}$ (Bennett 1984). In a nonlocal regime with $\alpha=3$, the structure function will scale like $r^{2}$. In a local regime with $\alpha=5 / 3$, the structure function will scale like $r^{2 / 3}$.

The longitudinal and transverse components of the structure function are not independent. Assuming that 




FIG. 3. Relative dispersion $D^{2}$ as a function of time, plotted (a) for the first 10 days on a semilog axis, and (b) the full record on log-log axes. The blue, green, and red curves denote the observed, AVISO-advected, and stochastic (AVISO-advected with stochastic closure) drifter trajectories, respectively. The shaded area is the bootstrapped $95 \%$ confidence interval of the relative dispersion. Theoretical power laws are indicated by black dotted lines.

the structure function has a power law dependence $S_{2} \sim r^{m}$, a purely nondivergent flow will lead to a ratio of $S_{t} / S_{l}=m+1$, and a purely irrotational flow will lead to $S_{t} / S_{l}=1 /(m+1)$ (Balwada et al. 2016).

\section{5) Helmholtz DeCOMPOSITION}

As for every vector field, we can decompose the velocity vector into divergent and rotational components $\mathbf{u}=-\nabla \times(\mathbf{k} \psi)+\nabla \phi$. Performing a Helmholtz decomposition of the velocity structure function (Bühler et al. 2014; Lindborg 2015), we can separate rotational $S_{r}$ and divergent $S_{d}$ components of the relative velocity and further characterize the flow field. We compute the divergent and rotational structure functions as

$$
\begin{aligned}
& S_{d}(r)=S_{l}(r)-\int_{0}^{r} \frac{1}{r}\left(S_{t}-S_{l}\right) d r, \\
& S_{r}(r)=S_{t}(r)+\int_{0}^{r} \frac{1}{r}\left(S_{t}-S_{l}\right) d r .
\end{aligned}
$$

In a two-dimensional, quasigeostrophic regime, we expect the flow to be nearly nondivergent. In this case the rotational component of the structure function will be $S_{r}>S_{d}$. In an unbalanced wave continuum, for example, with a Garrett-Munk spectrum, we expect the divergent component to become important. For internal waves the ratio of $S_{r} / S_{d}$ depends on wave frequency and inertial frequency (Bühler et al. 2014). Near-inertial oscillations are expected to have $S_{r} / S_{d}=1$. Furthermore, the divergent and rotational components help to identify the scales at which dynamics transition from one regime to another.

\section{Results}

In this section, we characterize the dispersion in the upper ocean using the pair dispersion statistics and structure functions described above. We focus on the early-time and small-scale behavior that falls into the spatiotemporal regime of submesoscale motions.

\section{a. Relative dispersion}

The observed relative dispersion $D^{2}$ calculated from the drifter array shows two distinct regimes (Fig. 3). During the first 4 days when separations are small $(r<10 \mathrm{~km})$, relative dispersion grows exponentially with an $e$-folding time scale of about 1.25 days. As separations reach the deformation radius $L_{D}=60 \mathrm{~km}$, they transition approximately to a $t^{3}$ power law. The initial exponential growth is as expected for nonlocal dispersion, as is the cubic power law growth for local dispersion.

At long times, $t>100$ days, relative dispersion does not flatten to linear growth. Linear growth is expected for constant relative dispersion if pair separations grow larger than the dominant eddy size $(\approx 100 \mathrm{~km})$ and become uncorrelated (Fig. 4). A possible explanation is that the Indian coast prevents drifters from spreading isotropically and drifters become entrained into the boundary current (Fig. 1). Furthermore, dispersion calculated for 


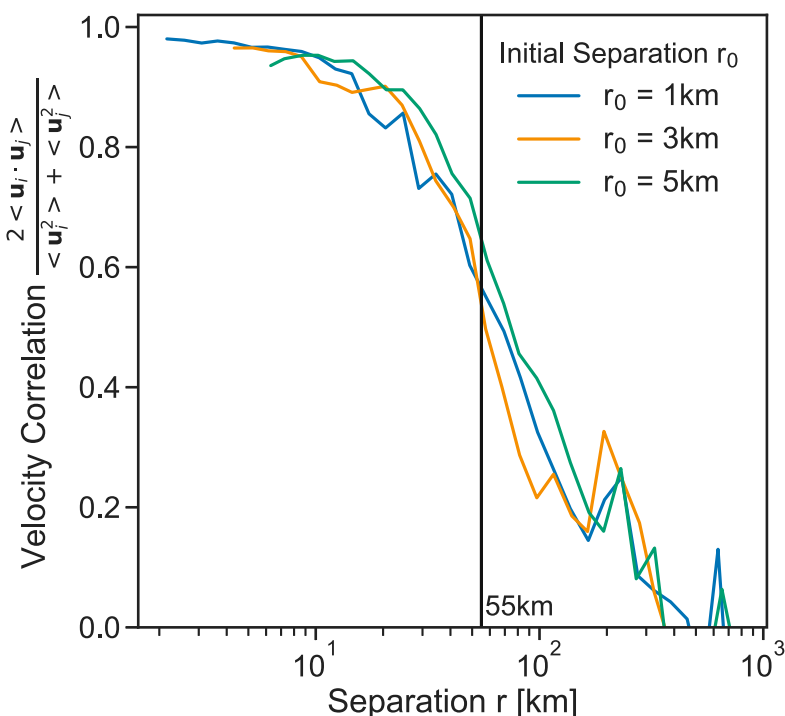

FIG. 4. Pair velocity correlation normalized by the single particle velocity variance for three different initial separations: $0 \leq r_{0}<2 \mathrm{~km}$ (blue), $2 \leq r_{0}<4 \mathrm{~km}$ (orange), $4 \leq r_{0}<6 \mathrm{~km}$ (green). The black line indicates an estimate for the decorrelation scale, where the correlation has fallen off by $1 / e$ from its maximum.

large separations above $100 \mathrm{~km}$ has uncertainty, as only a small number of drifter pairs is available at this scale.

The simulated, AVISO-advected drifters are explicitly advected with geostrophic currents that lack variability at scales smaller than $1 / 4^{\circ}$. AVISOadvected drifters are able to reflect the overall drift pattern of the observed drifters. Their trajectories, however, are significantly smoother and their rates of dispersion are slower than for the observed drifters. After the same period of drift, AVISO-advected drifters stay much more coherent and spread over a much smaller area than the observed drifter trajectories (Fig. 1).

Estimates of relative dispersion further illustrate the difference between the AVISO drifters and the observed drifters (Fig. 3). Since the AVISO energy spectrum is steep, likely $\alpha \gg 3$, nonlocal dispersion and exponential growth is expected. For the first 10 days, the AVISO relative dispersion grows exponentially, however, with a small growth parameter. Since the AVISOderived currents lack variability below $1 / 4^{\circ}$ (about $30 \mathrm{~km}$ ), the dispersion at small scales is dominated by mesoscale shear. After 10 days, however, the AVISO drifters approximately follow a $t^{3}$ power law. Interestingly, the AVISO drifters show the same plateau in relative dispersion as the observed drifters after about 5-10 days, possibly, a synoptic feature that traps drifters the same way as in the observations (Fig. 3b).

The stochastic drifters that include superposed noise in addition to the AVISO currents improve both the qualitative trajectories (Fig. 1), as well as the relative dispersion estimates. Generally, the stochastic noise increases the magnitude of relative dispersion compared to the AVISO drifters, such that the behavior is closer to the observed drifters. During the first 4 days, however, stochastic drifters show a different behavior than the observed drifters. Their relative dispersion grows more rapidly, possibly like $t^{2}$ or $t^{3}$. The late-time behavior is within $95 \%$ of the observed drifters and shows a $t^{2}$ power law. An alternative explanation for this behavior is the result of random motion in the mesoscale shear which produces the same relative dispersion as Richardson dispersion (Bennett 1987).

The relative diffusivity $\kappa_{\text {rel }}$ is noisier than the relative dispersion because it is the time derivative of relative dispersion. In our data, we locally encounter zero and negative slope of $D^{2}$, suggesting converging pairs of drifters for which the $\kappa_{\text {rel }}$ is not well defined. Here, we calculate the derivative of the mean square separation, focusing on diffusivity that is larger than zero and only using those values that consist of at least 20 data points.

In Fig. 5, the relative diffusivity is shown as a function of pair separation. For the observed drifters, a $r^{2}$ dependence is evident at separations below the deformation radius $L_{D}$. Although the variability is large, a transition is expected at $L_{D}$, where the relative diffusivity starts to flatten to a $r^{4 / 3}$ power law. As already pointed out in the relative dispersion results, the diffusivity does not saturate at the largest, observed scales suggesting that a regime of constant relative diffusivity is not reached. This would be expected if $r>L_{D}$ and the combined effect of multiple eddies is sampled. The theoretical expectations for nonlocal energy spectra $\alpha \geq 3$ is that the diffusivity grows like $r^{2}$. For a local energy spectrum with $1<\alpha \leq 3$, the theoretical prediction is $r^{(\alpha+1) / 2}$. For $\alpha=5 / 3$, specifically, the diffusivity grows like $r^{4 / 3}$, Richardson's 4/3 law. The observations lie in an envelope in between the local and nonlocal regime, curve fitting suggests, however, that it more closely follows a $r^{2}$ power law.

The AVISO drifters and stochastic drifters show a similar behavior but with lower overall diffusivity. The stochastic noise increases the diffusivity compared to the AVISO drifters which is particularly pronounced at small scales below $5 \mathrm{~km}$. Curve fitting results suggests that the AVISO drifters follow $r^{2}$ more closely, whereas the stochastic drifters follow $r^{4 / 3}$. At the largest scales, the stochastic and AVISO drifters have a diffusivity that is an order of magnitude smaller than the observed, despite the expectation that all three datasets should converge to the same diffusivity. This might be a result that is caused by a strong reduction of available pairs at these scales and the fact that the simulated drifter 


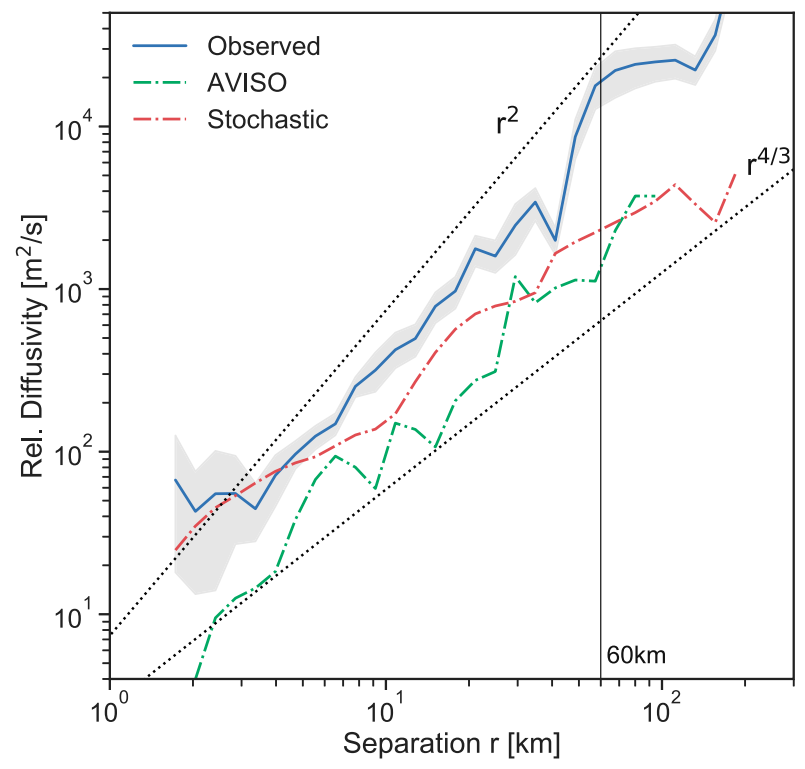

FIG. 5. Relative diffusivity, the time derivative of the relative dispersion (Fig. 3), as a function of separation $r$. The blue, green, and red curves denote the observed, AVISO-advected, and stochastic (AVISO-advected with stochastic closure) drifter trajectories, respectively. The shaded area is the bootstrapped $95 \%$ confidence interval of the relative diffusivity. The dotted lines indicate $r^{2}$ (expected in nonlocal regimes) and $r^{4 / 3}$ (expected in a local regime).

experiment might not have reached saturation over the duration of the observed drifter experiment.

\section{b. Finite-size Lyapunov exponent}

The observed FSLEs (Fig. 6) are largest for the smallest separations. The associated $e$-folding time is about one day which is close to the $e$-folding time estimated from the initial phase of the relative dispersion. Due to the limited number of pairs available, however, uncertainties are large at the smallest scales. With the number of data points available, it is difficult to validate, whether the FSLE is constant over a range of scales as predicted for exponential pair dispersion. At intermediate scales, below $L_{D}$, the FSLEs scale like $r^{-2 / 5}$. The equivalent slope of the relative dispersion is 5 , indicating a rapid time scale of separation. In agreement with the relative dispersion and diffusivity, we find a change of behavior at the deformation radius. For separations larger than $L_{D}$, FSLEs fall off like $r^{-2 / 3}$, which is the prediction for a Richardson-like dispersion with $D^{2} \sim t^{3}$.

The AVISO drifters show constant FSLEs from about $1 \mathrm{~km}$ to the deformation radius. If the FSLE is constant across a range of scales, the $e$-folding time is constant, and pairs separate exponentially fast. The smallest scales below $1 \mathrm{~km}$, however, suffer from large

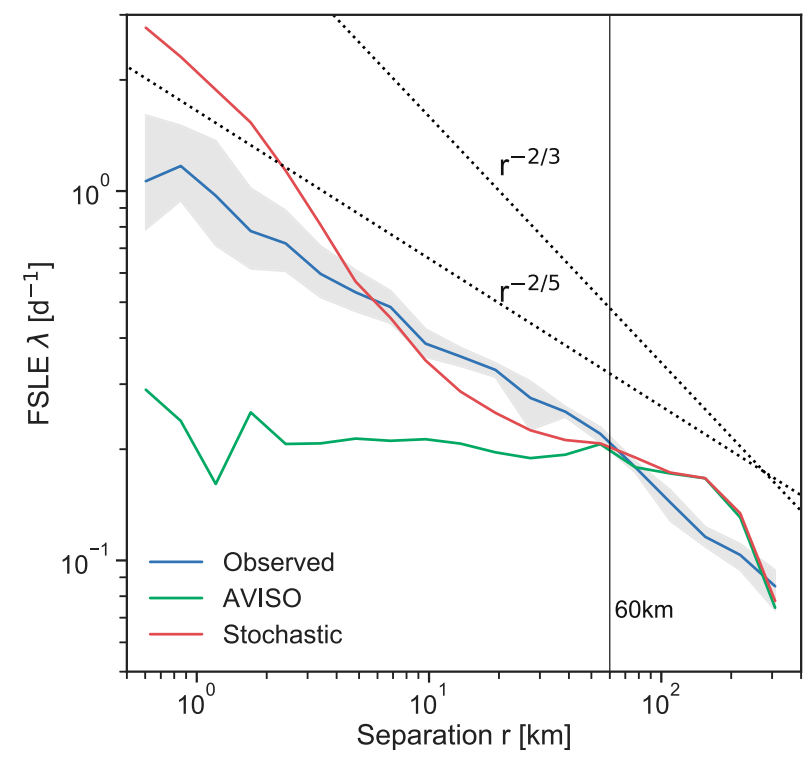

FIG. 6. FSLE as a function of separation $r$. The blue, green, and red curves denote the observed, AVISO-advected, and, stochastic (AVISO-advected with stochastic closure) drifters, respectively. The shaded area is the bootstrapped $95 \%$ confidence interval of the FSLE. Dotted lines denote power law dependencies.

uncertainty. Above $L_{D}$, the FSLEs fall off more slowly than for the observed drifters. The stochastic noise generates FSLEs that are larger than the observed FSLEs for scales from 0.1 to $10 \mathrm{~km}$. The effect of the noise decreases with increasing $r$, such that the stochastic drifters behave like the AVISO drifters above $L_{D}$. The large initial FSLEs suggest faster separation rates of the stochastic trajectories than the observed trajectories, which is in agreement with the relative dispersion results. Yet, this increase in separation rates is a function of the magnitude of the stochastic noise (section 2d).

Since time resolution is important in the computation of FSLEs and the sea level anomaly data is updated daily, it is expected that FSLE estimates from AVISOadvected drifters cannot resolve the $e$-folding time scale of 1-2 days below $5 \mathrm{~km}$. Similarly, the 30 -min resolution of the observed drifters might not be able to resolve the true maximum FSLE.

\section{c. Pair separation PDFs}

The pair separation PDFs (at $t=3$ days) are shown for three different initial separations in Fig. 7. We choose separation classes that are centered at $r_{0}=1,3$, and $5 \mathrm{~km}$, each of which are $2 \mathrm{~km}$ wide (e.g., for the first class $\left.0<r_{0}<2 \mathrm{~km}\right)$. These initial separations are selected because they are in the range or scales for which FSLEs are large and relative dispersion indicates exponential growth. Consider first the observed drifters (Fig. 7a). 

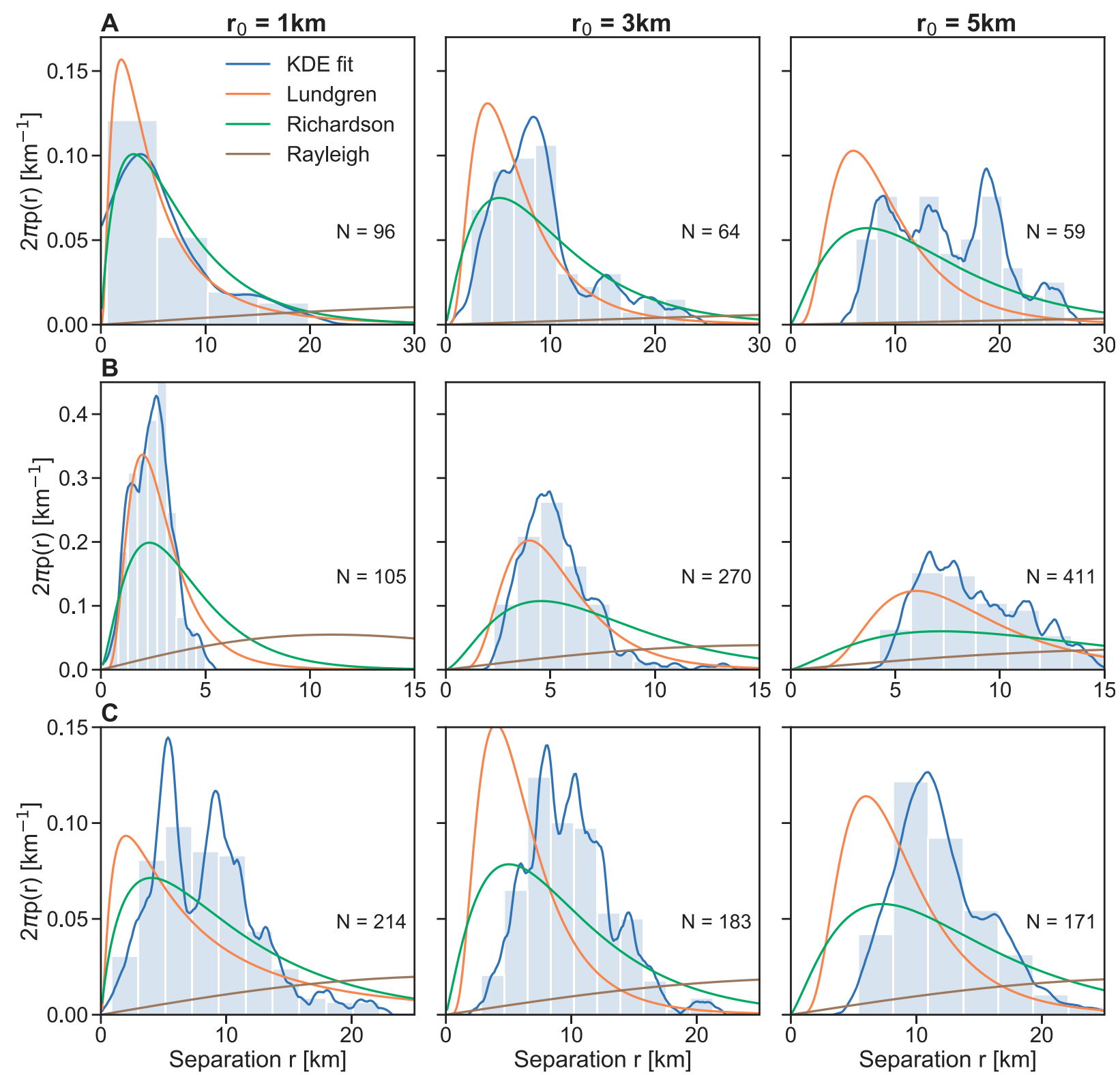

FIG. 7. Pair separation PDFs at $t=3$ days for different initial separations $r_{0}$ for each of the three datasets: (a) observed, (b) AVISOadvected, and (c) stochastic (AVISO-advected with stochastic closure) drifters. The theoretical PDFs are shown in color, for the nonlocal Lundgren (orange), local Richardson (green), and uncorrelated Rayleigh (brown) regimes. The histogram and kernel density estimation of the measured distributions is in blue.

Separations in the smallest separation class $\left(r_{0}=1 \mathrm{~km}\right)$ are associated with the most peaked PDF. The two larger initial separation classes $\left(r_{0}=3 \mathrm{~km}\right.$ and $\left.r_{0}=5 \mathrm{~km}\right)$ are less peaked. The theoretical PDFs whose parameters are estimated from the relative dispersion curves (see section 2 and Table 1 ) are calculated corresponding to the three initial separations $r_{0}$ at $t=3$ days. The (nonlocal) Lundgren regime and the (local) Richardson regime are both close to the observed data in the $1-\mathrm{km}$ class. The theoretical curve for the Richardson regime and the estimated curve from kernel density estimation of the observed data are not significantly different. The larger the initial separation, the lower the predictive power of the theoretical PDFs.

The PDFs for the AVISO separations (Fig. 7b) occupy a much smaller range of scales. It is evident that the Lundgren distribution is the best fit for all three initial separation classes. The stochastic drifters, however, behave differently from the two other datasets. The stochastic noise causes a fast widening of the PDFs, 
TABLE 1. Estimated parameters for the theoretical pair separation PDFs.

\begin{tabular}{|c|c|c|c|c|c|c|c|c|c|}
\hline \multirow[b]{2}{*}{ Separation $r_{0}(\mathrm{~km})$} & \multicolumn{3}{|c|}{ Observed } & \multicolumn{3}{|c|}{ AVISO } & \multicolumn{3}{|c|}{ Stochastic } \\
\hline & 1 & 3 & 5 & 1 & 3 & 5 & 1 & 3 & 5 \\
\hline$\kappa($ Rayleigh $)\left(\mathrm{km}^{2} \mathrm{day}^{-1}\right)$ & 398 & 793 & 1300 & 20 & 40 & 55 & 148 & 161 & 165 \\
\hline$T$ (Lundgren) $\left(\right.$ day $\left.^{-1}\right)$ & 7.7 & 15.2 & 19.6 & 22.2 & 30 & 26.1 & 4.7 & 18.8 & 22.9 \\
\hline$\beta$ (Richardson) $\left(\mathrm{km}^{2 / 3}\right.$ day $\left.^{-1}\right)$ & 0.51 & 0.41 & 0.52 & 0.2 & 0.28 & 0.40 & 0.74 & 0.45 & 0.51 \\
\hline
\end{tabular}

such that both Lundgren and Richardson solutions fail to predict the distribution. The widening of the PDF is caused by uncorrelated motions and is comparable to the Rayleigh dispersion. Since we estimate the diffusion coefficient from the late-time behavior ( $t>10$ days) of the relative dispersion, this diffusivity is not captured. The fast dispersion of the stochastic drifters at small scales $(r<10 \mathrm{~km})$ has also been observed in the relative diffusivity (Fig. 5) and the FSLE (Fig. 6). The character of this dispersion is different from Lundgren and Richardson as well as the late-time Rayleigh regime.

The theoretical solution for the Rayleigh regime, that is, dispersion by uncorrelated pair velocities, does not describe any of the datasets. This is expected for these small initial separations and after 3 days, in particular, because the diffusivity for the Rayleigh regimes is estimated from the late-time relative dispersion. As we see in the velocity cross correlation (Fig. 4), the velocities are correlated below the deformation radius $L_{D}$. Since all drifters are deployed in the same region, we expect that pairs likely sample the same features in all three datasets. Additionally, it is unclear if the diffusion coefficient $\kappa_{2}$ can be determined accurately from the relative dispersion curves for two reasons: 1) As the late-time behavior is covered by fewer pairs, the uncertainties are relatively large, and 2) the relative dispersion curve does not unambiguously reach the linear growth regimes that are expected for uncorrelated motions.

The kurtosis, the fourth moment of the PDFs, is a metric to quantify the peakedness of a distribution. We group the data into the three classes of initial separation $r_{0}$ and evaluate the kurtosis for each of those groups as a function of time (Fig. 8).

In case of the observed drifters (Fig. 8a), pairs that are initially close $\left(r_{0}=1 \mathrm{~km}\right)$ generate an exponentially growing kurtosis that reaches a maximum value of 32 (not shown), clearly suggesting nonlocal behavior of the dispersion. Pairs with larger initial separation grow more slowly and reach a smaller maximum kurtosis (yet larger than the maximum value $5.6 \mathrm{ex}$ pected for the Richardson regime). Interestingly, the kurtosis quickly falls off to values between 1 and 5 after about 15 days. It is somewhat surprising that values decrease so quickly, given that the energy-containing eddy size is clearly mesoscale and $\mathscr{O}(60) \mathrm{km}$. A possible explanation for this is the sensitivity of the kurtosis to the tails of the pair separation PDFs. Pairs in the tails of the distribution develop quickly and could decrease the kurtosis.

The AVISO drifters (Fig. 8b) produce less of an exponential growth (at least at these initial separation scales) with maximum values that are larger than 5.6. The late-time asymptotic behavior of the AVISO


FIG. 8. Kurtosis of the pair separation PDFs as a function of time for the (a) observed, (b) AVISO-advected, and (c) stochastic drifters (AVISO-advected with stochastic closure). Colors indicate the three classes of initial pair separation $r_{0}$. Dotted lines indicate the values expected in the asymptotic limit of the Lundgren regime $\left(\sim e^{t}\right)$, the Richardson regime (5.6), and the Rayleigh regime (2). 



FIG. 9. (a) Second-order velocity structure functions as a function of separation $r$ for the observed, AVISOadvected, stochastic (AVISO-advected with stochastic closure), and low-pass-filtered trajectories. (b) Velocity structure functions after decomposition into longitudinal and transverse, and rotational and divergent components. The total observed structure function is the same in (a) and (b). The solid vertical line indicates the length scale at which the rotational component becomes dominant. The dotted lines indicate the theoretical slopes of nonlocal $\left(S_{2} \sim r^{2}\right)$, local $\left(S_{2} \sim r^{2 / 3}\right)$, and frontal energy spectra $\left(S_{2} \sim r^{1}\right)$.

drifters could be identified as Richardson-like, as values oscillate around 5.6. Interestingly, and opposed to the observed drifters, values are larger than 2 , suggesting that a Rayleigh regime is unlikely.

Contrary to the AVISO drifters, the stochastic drifters generate an exponentially growing kurtosis (Fig. 8c). Particularly the smallest separation class grows to a maximum value of 25 , which can clearly be attributed to nonlocal behavior. The difference between AVISO drifters and stochastic drifters suggests that the stochastic noise facilitates faster separation. The exponential growth of the kurtosis also occurs at a later time than for the observed drifters which is an artifact of the resolution of the AVISO currents.

\section{d. Structure functions}

The structure functions $S_{2}$ for the observed drifters are shown in Fig. 9a as a function of separation $r$. At intermediate scales, $10-100 \mathrm{~km}, S_{2}$ approximately follows a $r^{2 / 3}$ power law. From scaling arguments, we know that $S_{2} \sim r^{2 / 3}$ is the expectation for an energy spectrum with $E \sim k^{-5 / 3}$. At small scales $(r<10 \mathrm{~km})$ and at large scales $(r>100 \mathrm{~km}), S_{2}$ flattens to $r^{1 / 3}$ and $r^{1 / 2}$, respectively.

Low-pass filtering the trajectories affects $S_{2}$ such that it has less energy than the unfiltered dataset below $100 \mathrm{~km}$. It can be argued that the filtered $S_{2}$ steepens relative to the unfiltered dataset between 10 and $100 \mathrm{~km}$. In this range of scales, the power law is closer to $r^{1}$, which would be predicted for an energy spectrum of $E \sim k^{-2}$. As expected, the structure functions for the AVISO and stochastic drifters are smaller at scales below the resolution of the AVISO-derived currents (Fig. 9a). The $S_{2}$ from the AVISO drifters clearly follows a $r^{2}$ power law from 1 to $100 \mathrm{~km}$ and flattens above. This is expected since the energy spectrum for the AVISO currents $E \sim k^{-\alpha}$ is steep with $\alpha>3$. The noise in the velocity of the stochastic drifters causes $S_{2}$ to be flat at scales below $30 \mathrm{~km}$. Above that, $S_{2}$ from the stochastic drifters behaves similarly to $S_{2}$ from the AVISO drifters.

It is evident in Fig. 9b that the rotational component and the divergent component cross at approximately $75 \mathrm{~km}$, which is close to the local deformation radius $L_{D}=60 \mathrm{~km}$. The $S_{d}$ is dominant below $75 \mathrm{~km}$ and approximately determines the slope of $S_{2}$. Likewise, $S_{r}$ is dominant above $75 \mathrm{~km}$ and approximately determines the slope of $S_{2}$ at large scales. The longitudinal and transverse components also cross but at slightly smaller scales (about $50 \mathrm{~km}$ ). The $S_{l}$ is dominant below $50 \mathrm{~km}$, and $S_{t}$ becomes dominant above. The $S_{l}$ grows like $r^{2 / 3}$ up to $50 \mathrm{~km}$, consistent with a local energy spectrum, and flattens above.

The ratio of transverse to longitudinal components $S_{t} / S_{l}$ (Fig. 10a) of the observed drifters is constant at about 0.9 for $r<50 \mathrm{~km}$ and increases to about 1.5 for $r>75 \mathrm{~km}$. The ratio $S_{t} / S_{l}$ behaves the same way for the filtered drifter, while generally being larger. The AVISO drifters, however, generate a $S_{t} / S_{l}$ that is 

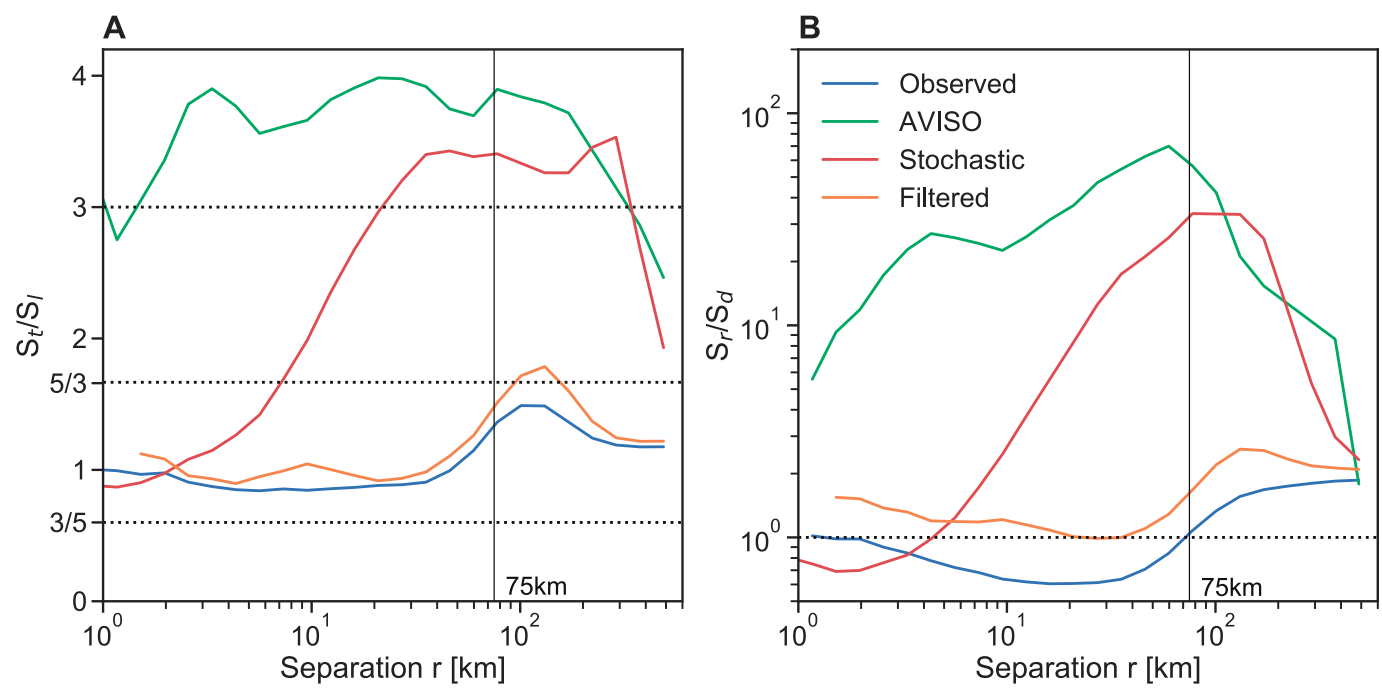

FIG. 10. (a) Ratio of the transverse and longitudinal component of the velocity structure function $\left(S_{t} / S_{l}\right)$ as a function of separation $r$. (b) Ratio of the rotational and divergent component of the velocity structure function after Helmholtz decomposition $\left(S_{r} / S_{d}\right)$ as a function of separation $r$. Colors denote the observed, AVISO-advected, stochastic (AVISOadvected with stochastic closure), and low-pass-filtered datasets. Horizontal dotted lines are for reference.

larger than 3 . The ratio $S_{t} / S_{l}$ for the stochastic drifters increases from 1 at the smallest scales to about 3.2 above $75 \mathrm{~km}$.

The ratio of rotational to divergent component $S_{r} / S_{d}$ (Fig. 10b) confirms the general pattern evident in the components of the structure function. The ratio $S_{r} / S_{d}$ for observed drifters is dominated by the rotational component for $r>75 \mathrm{~km}$. Filtering removes energy predominantly from the divergent component and shifts $S_{r} / S_{d}$ to higher values. It appears that $S_{r} / S_{d}$ for the filtered drifters is greater or equal to 1 at all times. In the cases of the AVISO drifters and the stochastic drifters, the rotational component is larger than the divergent component by at least an order of magnitude. At small scales, however, the divergent, stochastic noise dominates the stochastic $S_{r} / S_{d}$.

In the limit of divergence-dominated flow $(r<10 \mathrm{~km})$, the ratios agree with the theoretical expectations. The total structure function of the observed drifters has a slope of $m=1 / 3$ or flatter (Fig. 9a) which would predict $S_{t} / S_{l}$ to be about 3/4. Similarly, in the limit of rotation-dominated flow, the total structure function has a slope of $m=1 / 2$ which would predict $S_{t} / S_{l}$ to be 1.5 . For the AVISO field, for which $S_{r} / S_{d}>1$ at all scales, the total structure function has a slope of $m=2$ which predicts $S_{t} / S_{l}$ to be 3 .

The finding that motions are divergent at small scales suggests that two-dimensional, geostrophic dynamics are no longer dominant. Candidates for divergent motions could be the internal wave field, inertial oscillations, and Langmuir turbulence as well as the horizontally divergent submesoscale flow.

\section{Discussion}

\section{a. Dispersion regimes}

The results of the dispersion statistics (relative dispersion and diffusivity, FSLE, pair separation PDFs, and kurtosis) describe the dispersion characteristics of the flow in the Bay of Bengal. The combination of distance-averaged and time-averaged metrics allows for a more complete description of the flow. Additionally, the structure functions contribute to the understanding of the distribution of energy across scales. In the absence of knowledge about the energy spectra, this can be useful despite the fact that structure function can be affected by nondispersive modes, which are part of the true, observed flow.

The drifter-derived relative dispersion and FSLE (for pairs with $r_{0}<3 \mathrm{~km}$ ) agree qualitatively on small scales and at the deformation radius $L_{D}$ (Table 2). These suggests a nonlocal regime below $20 \mathrm{~km}$ and a local regime above the deformation radius $L_{D}$. However, the metrics disagree quantitatively: The $e$-folding time scale predicted by the FSLE is half as large as for the relative dispersion and the regime shift from exponential to power-law growth is at $20 \mathrm{~km}$ (Fig. 6) as opposed to $10 \mathrm{~km}$ shown in the relative dispersion (Fig. 3). The relative diffusivity is consistent with this result and grows like $r^{2}$, however, the transition from nonlocal to local is less pronounced. The general result agrees with previous results (Koszalka et al. 2009; LaCasce and Ohlmann 2003). The difference between FSLE and relative dispersion can be attributed to the 
TABLE 2. Dispersion regimes in Lagrangian statistics at submesoscales, $\mathscr{O}(0.1-10) \mathrm{km}$.

\begin{tabular}{lccc}
\hline & Richardson (local) & Lundgren (nonlocal) & Observed \\
\hline$E(k)$ & $k^{-5 / 3}$ & $k^{-3}$ & - \\
$S_{2}(r)$ & $r^{2 / 3}$ & $r^{2}$ & $r^{2 / 3}$ \\
$\lambda(r)$ & $r^{-2 / 3}$ & const & $r^{-2 / 3}$ \\
$D^{2}(t)$ & $t^{3}$ & $e^{t}$ & $e^{t}$ \\
$\kappa_{\text {rel }}(r)$ & $r^{4 / 3}$ & $r^{2}$ & $r^{2}$ \\
$\operatorname{kur}(t)$ & 5.6 & $e^{t}$ & $e^{t}$ \\
\hline
\end{tabular}

quality of the data (particularly the position error) (Haza et al. 2014; Poje et al. 2010) but can also be affected by the fact that different subsets of pairs are used in the calculation (LaCasce and Bower 2000).

The pair separation PDFs and their kurtoses suggest that a Lundgren distribution is the best fit for initially close pairs $\left(r_{0}=1 \mathrm{~km}\right)$ and for the first 10 days of the drift (Table 2). The rate of strain field of the mesoscale eddies is a logical candidate for the nonlocal dispersion of small drifters pairs. Furthermore, the kurtosis of the pair separation PDFs grows exponentially in time for small initial separations and for the first 10 days. The large kurtoses are a clear indication for nonlocal pair dispersion. Larger initial separations $\left(r_{0}>5 \mathrm{~km}\right)$, however, are well described by the Richardson solution to the PDF and kurtosis. The kurtosis suggests that the pair separations are uncorrelated for pairs that are larger than $5 \mathrm{~km}$. This is a much smaller length scale than the dominant eddy size.

On the contrary, structure functions show a $r^{2 / 3}$ power law for separation distances below the deformation radius $L_{D}$, suggesting a $k^{-5 / 3}$ energy spectrum and local dispersion (Table 2). The local character of the energy spectrum inferred from structure functions also includes motions that do not affect pair dispersion like internal waves (Babiano et al. 1990). Even after filtering inertial oscillations with a low-pass filter, however, structure functions indicate a local regime and do not steepen significantly. This findings is different from results in the Gulf of Mexico (Beron-Vera and LaCasce 2016), where the removal of near-inertial energy caused structure functions to steepen from an apparent $r^{2 / 3}$ power law to $r^{2}$, which is in line with the pair dispersion statistics.

The decomposition of structure functions indicates that motions are divergent at scales below $75 \mathrm{~km}$ and the ratio between rotational and divergent motions $S_{r} / S_{d}$ is less than one. These divergent motions can be attributed to the internal wave field as well as to ageostrophic submesoscale flows that would have an energy spectrum of $k^{-2}$ or shallower. However, the hypothesized transition from local to nonlocal dispersion at the submesoscale cannot be supported with the data available here. Neither the dispersion statistics (relative dispersion, diffusivity, FSLE, PDFs), nor the structure functions, seem to reflect a regime change at the mixed layer deformation radius estimated at $5-10 \mathrm{~km}$. Pair dispersion statistics as well as structure functions consistently indicate a regime shift close to the deformation radius $L_{D}=60 \mathrm{~km}$.

Energy at small scales that causes the structure function to indicate a local regime can likely not be explained by inertial oscillations, which begs the question as to which processes are responsible for the variability at those scales. We find that motions become increasingly divergent below the deformation radius and clearly deviate from two-dimensional, quasigeostrophic dynamics below $20 \mathrm{~km}$. The kurtosis supports this at scales above $5 \mathrm{~km}$, which quickly falls off to a value of 2 (as the Rayleigh asymptotic limit). The ratios between longitudinal and transverse structure functions, and between rotational and divergent structure functions, further suggest that those motions are weakly rotational, a property that applies to the internal wave continuum as well as to balanced dynamics that have a considerable ageostrophic component.

\section{b. Consequences of stochastic noise}

While at global or basin scale, AVISO-advected drifters can reproduce the observed relative dispersion, they are not sufficient to model dispersion on the regional scale, $\mathscr{O}(10) \mathrm{km}$. Our results suggest that the mesoscale circulation dominates pair dispersion and is therefore qualitatively captured by the AVISO-derived, geostrophic currents. However, small-scale perturbations, even if they are in the form of a simple first order stochastic model, drastically alter trajectories. The firstorder stochastic trajectories are a better representation of the observed trajectories and can reproduce the long-time dispersive behavior in the relative dispersion and FSLE.

The first-order model implemented on the AVISOadvected trajectories has two primary effects, both of which are illuminating when interpreting the dispersion of observed drifters. First, diffusive growth tends to be faster than exponential spreading at early times. The dominant drivers for pair separations are, thus, the uncorrelated motions due to the stochasticity in the velocity. This can most clearly be seen in the pair separation PDFs that resemble dispersion due to uncorrelated velocities (Fig. 7), the diffusive growth of the relative dispersion (Fig. 3) as well as the flattening of structure functions at the smallest scales. Second, random motion in a constant shear flow leads to a relative dispersion that grows like $t^{3}$. The added noise on the velocity of the stochastic drifters can cause them to disperse in a shear dispersion manner. Since the small-scale PDFs seem to indicate Rayleigh-type distributions but the relative dispersion and diffusivity 
suggest $t^{3}$ growth, shear dispersion is a likely candidate for the quick dispersion of the stochastic drifters.

The subgrid-scale noise affects scales much larger than the noise scale. In fact, FSLE and structure functions suggest that the stochastic noise affects drifter motions at scales up to $10 \mathrm{~km}$. This scale can be identified by comparing the stochastic drifters with the AVISO drifters. Haza et al. (2014) found that uncertainty in the drifter position can affect the dispersion statistics at scales up to 6 times the magnitude of the position error.

\section{c. Limitations of the dataset}

The results have to be interpreted taking into account the shortcomings of each metric and the limitations of the dataset itself. Since drifters were drogued at $15-\mathrm{m}$ depth, mixed layers were possibly shallower than that during the first month (Hormann et al. 2016), and submesoscale turbulence decays away from the surface, we expect that the drifters measure less small-scale variability and steeper spectra than they would at the very surface. The fact that the drogue is deeper than the mixed layer, furthermore, reduces the bias due to surface wave motions, and most likely reduces the effect of convergent flow such as Langmuir circulation on the distribution of drifters. These convergent flows otherwise tend to align drifters and bias the regions they sample.

Additionally, a sampling bias occurs because of the choice of deployment site. Certain features in the flow are sampled more extensively, rather than a representative sampling of the velocity field. The release location causes drifters to be entrained in a cyclonic eddy for the first 10 days. During this period, drifters traveled as a coherent cluster, reducing the degrees of freedom of our statistics due to dependent pairs.

The energy at the mesoscale might also overshadow any coherent small-scale motion and result in nonlocal dispersion. This has severe consequences when inferring the energy spectrum from dispersion statistics. Additionally, as our results suggest, there is a large uncertainty associated with the largest scales. At large scales, a limited number of drifters samples the mesoscale eddies, providing only a limited number of realizations.

\section{Conclusions}

The dispersion study presented here identifies a contradiction between pair dispersion statistics (relative dispersion, FSLE, pair separation PDFs) and an analysis of structure functions. Pair dispersion statistics consistently identify a nonlocal dispersion regime at scales below $20 \mathrm{~km}$ that is associated with an exponential rate of pair separation, and predicted by energy spectra that follow a $k^{-3}$ behavior. Structure functions, however, and their decomposition, suggest a local regime at scales below the deformation radius, associated with a power law growth of pair separations and predicted by energy spectra that are $k^{-5 / 3}$ or flatter. This discrepancy can be explained by 1) processes that contribute to the energy spectrum of a flow, but not significantly to the dispersion, or 2) uncertainties at the smallest scales, such as uncertainties in the position data.

To answer the question we posed in the title, submesoscale flows are possible to observe from pair dispersion statistics, however, the interpretation of the data can be difficult due to the richness of processes occupying the same spatiotemporal band. In particular, we find that there are motions such as near-inertial oscillations and tides that affect the energy spectrum, but are inefficient at dispersion. Theoretical predictions for the submesoscale range, therefore, do not hold for the observed data. The limitations of our dataset further constrains our ability to resolve the submesoscale range.

Our findings are relevant when studying drifter dispersion at submesoscales, especially in the presence of an energetic mesoscale circulation, as they question the ability of pair dispersion statistics to capture submesoscale flows. Alternatively, in a region of thin mixed layers, submesoscale flows could be inefficient at dispersing drifters as there is less available potential energy to be extracted by mixed layer instabilities.

Since dispersion statistics are often not conclusive in inferring an energy spectrum from pair statistics, particularly at small scales, more information about the flow field is needed. Multiple drifter statistics and clusters can help to map velocity gradients to further characterize the kinematics of a flow field. Velocity gradients are especially important in submesoscale flows, where the local Rossby number becomes $\mathscr{O}(1)$. Analyses of multiple drifters have not been fully exploited in oceanography despite their potential, often due to a lack of suitable clustered experiments.

Acknowledgments. This research was supported by the Air Sea Interaction Regional Initiative (ASIRI) under ONR Grant N00014-13-1-0451 (SE and AM) and ONR Grant N00014-13-1-0477 (VH and LC). Additionally, AM and SE thank NSF (Grant OCEI434788) and ONR (Grant N00014-16-1-2470) for support; VH and LC were further supported by ONR Grant N00014-15-1-2286 and NOAA GDP Grant NA10OAR4320156. We thank Joe LaCasce, Dhruv Balwada, and one anonymous reviewer for helpful comments and discussions that significantly improved this manuscript. The authors thank the captain and crew of the R/V Roger Revelle. The SVP-type drifters are part of the Global Drifter Program and supported by ONR 
Grant N00014-15-1-2286 and NOAA GDP Grant NA10OAR4320156 and are available under http:// www.aoml.noaa.gov/phod/dac/. The Ssalto/Duacs altimeter products were produced and distributed by the Copernicus Marine and Environment Monitoring Service (CMEMS, http://www.marine.copernicus.eu).

\section{REFERENCES}

Artale, V., G. Boffetta, A. Celani, M. Cencini, and A. Vulpiani, 1997: Dispersion of passive tracers in closed basins: Beyond the diffusion coefficient. Phys. Fluids, 9, 3162-3171, https:// doi.org/10.1063/1.869433.

Aurell, E., G. Boffetta, A. Crisanti, G. Paladin, and A. Vulpiani, 1997: Predictability in the large: An extension of the concept of Lyapunov exponent. J. Phys. A.: Math. Gen., 30, 1-26, https://doi.org/10.1088/0305-4470/30/1/003.

Babiano, A., C. Basdevant, P. Le Roy, and R. Sadourny, 1990: Relative dispersion in two-dimensional turbulence. J. Fluid Mech., 214, 535-557, https://doi.org/10.1017/S0022112090000258.

Balwada, D., J. H. LaCasce, and K. G. Speer, 2016: Scaledependent distribution of kinetic energy from surface drifters in the Gulf of Mexico. Geophys. Res. Lett., 43, 10 856-10863, https://doi.org/10.1002/2016GL069405.

Bennett, A. F., 1984: Relative dispersion: Local and nonlocal dynamics. J. Atmos. Sci., 41, 1881-1886, https://doi.org/10.1175/ 1520-0469(1984)041<1881:RDLAND>2.0.CO;2.

_ 1987: A Lagrangian analysis of turbulent diffusion. Rev. Geophys., 25, 799-822, https://doi.org/10.1029/RG025i004p00799.

Bennett, A., 2006: Lagrangian Fluid Dynamics. Cambridge University Press, $310 \mathrm{pp}$.

Beron-Vera, F. J., and J. H. LaCasce, 2016: Statistics of simulated and observed pair separations in the Gulf of Mexico. J. Phys. Oceanogr., 46, 2183-2199, https://doi.org/10.1175/JPO-D-15-0127.1.

Boccaletti, G., R. Ferrari, and B. Fox-Kemper, 2007: Mixed layer instabilities and restratification. J. Phys. Oceanogr., 37, 2228 2250, https://doi.org/10.1175/JPO3101.1.

Bühler, O., J. Callies, and R. Ferrari, 2014: Wave-vortex decomposition of one-dimensional ship-track data. J. Fluid Mech., 756, 1007-1026, https://doi.org/10.1017/jfm.2014.488.

Callies, J., and R. Ferrari, 2013: Interpreting energy and tracer spectra of upper-ocean turbulence in the submesoscale range (1-200 km). J. Phys. Oceanogr., 43, 2456-2474, https://doi.org/ 10.1175/JPO-D-13-063.1.

,- — J. M. Klymak, and J. Gula, 2015: Seasonality in submesoscale turbulence. Nat. Commun., 6, 6862, https://doi.org/ 10.1038/ncomms7862.

Centurioni, L. R., 2018: Drifter technology and impacts for sea surface temperature, sea-level pressure, and ocean circulation studies. Observing the Oceans in Real Time, R. Venkatesan et al., Eds., Springer, 37-57.

—, A. Horányi, C. Cardinali, E. Charpentier, and R. Lumpkin, 2017: A global ocean observing system for measuring sea level atmospheric pressure: Effects and impacts on numerical weather prediction. Bull. Amer. Meteor. Soc., 98, 231-238, https://doi.org/10.1175/BAMS-D-15-00080.1.

Charney, J. G., 1971: Geostrophic turbulence. J. Atmos. Sci., 28, 1087-1095, https://doi.org/10.1175/1520-0469(1971) $028<1087: \mathrm{GT}>2.0 . \mathrm{CO} ; 2$.

Chelton, D. B., R. A. deSzoeke, M. G. Schlax, K. E. Naggar, and N. Siwertz, 1998: Geographical variability of the first baroclinic Rossby radius of deformation. J. Phys. Oceanogr., 28, 433-460, https://doi.org/10.1175/1520-0485(1998) 028<0433:GVOTFB > 2.0.CO;2.

D'Asaro, E. A., and Coauthors, 2018: Ocean convergence and the dispersion of flotsam. Proc. Natl. Acad. Sci. USA, 115, 11621167, https://doi.org/10.1073/pnas.1718453115.

Ducet, N., P. Y. Le Traon, and G. Reverdin, 2000: Global highresolution mapping of ocean circulation from TOPEX/ Poseidon and ERS-1 and -2. J. Geophys. Res., 105, 19477-19498, https://doi.org/10.1029/2000JC900063.

Er-El, J., and R. L. Peskin, 1981: Relative diffusion of constantlevel balloons in the Southern Hemisphere. J. Atmos. Sci., 38, 2264-2274, https://doi.org/10.1175/1520-0469(1981)038<2264: RDOCLB $>2.0 . C O ; 2$.

Foussard, A., S. Berti, X. Perrot, and G. Lapeyre, 2017: Relative dispersion in generalized two-dimensional turbulence. J. Fluid Mech., 821, 358-383, https://doi.org/10.1017/jfm.2017.253.

Fox-Kemper, B., R. Ferrari, and R. Hallberg, 2008: Parameterization of mixed layer eddies. Part I: Theory and diagnosis. J. Phys. Oceanogr., 38, 1145-1165, https://doi.org/10.1175/2007JPO3792.1.

Gon, Y. J., K. S. Yong, and K. H. Seong, 2018: Spectral descriptions of submesoscale surface circulation in a coastal region. J. Geophys. Res. Oceans, 123, 4224-4249, https:// doi.org/10.1029/2016JC012517.

Graff, L. S., S. Guttu, and J. H. LaCasce, 2015: Relative dispersion in the atmosphere from reanalysis winds. J. Atmos. Sci., 72, 2769-2785, https://doi.org/10.1175/JAS-D-14-0225.1.

Griffa, A., K. Owens, L. Piterbarg, and B. Rozovskii, 1995: Estimates of turbulence parameters from Lagrangian data using a stochastic particle model. J. Mar. Res., 53, 371-401, https:// doi.org/10.1357/0022240953213151.

Haller, G., 2015: Lagrangian coherent structures. Annu. Rev. Fluid Mech., 47, 137-162, https://doi.org/10.1146/annurev-fluid010313-141322.

Haza, A. C., A. C. Poje, T. M. Özgökmen, and P. Martin, 2008: Relative dispersion from a high-resolution coastal model of the Adriatic Sea. Ocean Modell., 22, 48-65, https://doi.org/ 10.1016/j.ocemod.2008.01.006.

— , T. M. Özgökmen, A. Griffa, Z. D. Garraffo, and L. Piterbarg, 2012: Parameterization of particle transport at submesoscales in the Gulf Stream region using Lagrangian subgridscale models. Ocean Modell., 42, 31-49, https://doi.org/10.1016/ j.ocemod.2011.11.005.

,,,-- A. C. Poje, and M.-P. Lelong, 2014: How does drifter position uncertainty affect ocean dispersion estimates? J. Atmos. Oceanic Technol., 31, 2809-2828, https://doi.org/ 10.1175/JTECH-D-14-00107.1.

Horányi, A., C. Cardinali, and L. Centurioni, 2017: The global numerical weather prediction impact of mean-sea-level pressure observations from drifting buoys. Quart. J. Roy. Meteor. Soc., 143, 974-985, https://doi.org/10.1002/qj.2981.

Hormann, V., L. Centurioni, A. Mahadevan, S. Essink, E. D'Asaro, and B. P. Kumar, 2016: Variability of near-surface circulation and sea surface salinity observed from Lagrangian drifters in the northern Bay of Bengal during the waning 2015 Southwest Monsoon. Oceanography, 29 (2), 124-133, https:// doi.org/10.5670/oceanog.2016.45.

Hoskins, B. J., 1982: The mathematical theory of frontogenesis. Annu. Rev. Fluid Mech., 14, 131-151, https://doi.org/10.1146/ annurev.fl.14.010182.001023.

Jaeger, G. S., and A. Mahadevan, 2018: Submesoscale-selective compensation of fronts in a salinity-stratified ocean. Sci. Adv., 4, e1701504, https://doi.org/10.1126/sciadv.1701504. 
Kirwan, A. D., G. J. McNally, E. Reyna, and W. J. Merrell, 1978: The near-surface circulation of the eastern North Pacific. J. Phys. Oceanogr., 8, 937-945, https://doi.org/10.1175/1520-0485(1978) $008<0937$ :TNSCOT $>2.0$.CO;2.

Koszalka, I., J. H. LaCasce, and K. A. Orvik, 2009: Relative dispersion in the Nordic Seas. J. Mar. Res., 67, 411-433, https:// doi.org/10.1357/002224009790741102.

Kraichnan, R. H., 1967: Inertial ranges in two-dimensional turbulence. Phys. Fluids, 10,1417, https://doi.org/10.1063/1.1762301.

LaCasce, J. H., 2008: Statistics from Lagrangian observations. Prog. Oceanogr., 77, 1-29, https://doi.org/10.1016/j.pocean.2008.02.002.

- 2010 : Relative displacement probability distribution functions from balloons and drifters. J. Mar. Res., 68, 433-457, https://doi.org/10.1357/002224010794657155.

—_, 2016: Estimating Eulerian energy spectra from drifters. Fluids, 1, 33, https://doi.org/10.3390/fluids1040033.

, and A. Bower, 2000: Relative dispersion in the subsurface North Atlantic. J. Mar. Res., 58, 863-894, https://doi.org/ 10.1357/002224000763485737.

— , and C. Ohlmann, 2003: Relative dispersion at the surface of the Gulf of Mexico. J. Mar. Res., 61, 285-312, https://doi.org/ 10.1357/002224003322201205.

Lapeyre, G., and P. Klein, 2006: Dynamics of the upper oceanic layers in terms of surface quasigeostrophy theory. J. Phys. Oceanogr., 36, 165-176, https://doi.org/10.1175/JPO2840.1.

Le Traon, P. Y., F. Nadal, and N. Ducet, 1998: An improved mapping method of multisatellite altimeter data. J. Atmos. Oceanic Technol., 15, 522-534, https://doi.org/10.1175/ 1520-0426(1998)015<0522:AIMMOM > 2.0.CO;2.

Lin, J.-T., 1972: Relative dispersion in the enstrophy-cascading inertial range of homogeneous two-dimensional turbulence. J. Atmos. Sci., 29, 394-396, https://doi.org/10.1175/15200469(1972)029<0394:RDITEC > 2.0.CO;2.

Lindborg, E., 2015: A Helmholtz decomposition of structure functions and spectra calculated from aircraft data. J. Fluid Mech., 762, R4, https://doi.org/10.1017/jfm.2014.685.

Lucas, A. J., and Coauthors, 2014: Mixing to monsoons: Air-sea interactions in the Bay of Bengal. Eos, Trans. Amer. Geophys. Union, 95, 269-270, https://doi.org/10.1002/2014EO300001.

Lumpkin, R., and S. Elipot, 2010: Surface drifter pair spreading in the North Atlantic. J. Geophys. Res., 115, C12017, https:// doi.org/10.1029/2010JC006338.

Lundgren, T. S., 1981: Turbulent pair dispersion and scalar diffusion. J. Fluid Mech., 111, 27-57, https://doi.org/10.1017/ S0022112081002280.

Mahadevan, A., G. Spiro Jaeger, M. Freilich, M. M. Omand, E. Shroyer, and D. Sengupta, 2016: Freshwater in the Bay of Bengal: Its fate and role in air-sea heat exchange. Oceanography, 29 (2), 72-81, https://doi.org/10.5670/oceanog.2016.40.

Maximenko, N., R. Lumpkin, and L. Centurioni, 2013: Ocean surface circulation. Ocean Circulation and Climate: A 21st Century Perspective, G. Siedler et al., Eds., Elsevier, 283-304.

Morel, P., and M. Larceveque, 1974: Relative dispersion of constant-level balloons in the 200-mb general circulation. J. Atmos. Sci., 31, 2189-2196, https://doi.org/10.1175/15200469(1974)031<2189:RDOCBI>2.0.CO;2.

Nastrom, G. D., W. H. Jasperson, and K. S. Gage, 1986: Horizontal spectra of atmospheric tracers measured during the Global Atmospheric Sampling Program. J. Geophys. Res., 91, 13 20113 209, https://doi.org/10.1029/JD091iD12p13201.

Niiler, P. P., 2001: The world ocean surface circulation. Ocean Circulation and Climate: Observing and Modelling the Global Ocean, G. Siedler et al., Eds., Elsevier, 193-204.
— A. S. Sybrandy, K. Bi, P. M. Poulain, and D. Bitterman, 1995: Measurements of the water-following capability of holey-sock and TRISTAR drifters. Deep-Sea Res. I, 42, 1951-1955, https://doi.org/10.1016/0967-0637(95)00076-3.

Poje, A. C., A. C. Haza, T. M. Özgökmen, M. G. Magaldi, and Z. D. Garraffo, 2010: Resolution dependent relative dispersion statistics in a hierarchy of ocean models. Ocean Modell., 31, 36-50, https://doi.org/10.1016/j.ocemod.2009.09.002.

_ _, and Coauthors, 2014: Submesoscale dispersion in the vicinity of the Deepwater Horizon spill. Proc. Natl. Acad. Sci. USA, 111, 12 693-12 698, https://doi.org/10.1073/pnas.1402452111.

Ramachandran, S., and Coauthors, 2018: Submesoscale processes at shallow salinity fronts in the Bay of Bengal: Observations during the winter monsoon. J. Phys. Oceanogr., 48, 479-509, https://doi.org/10.1175/JPO-D-16-0283.1.

Richardson, L. F., 1926: Atmospheric diffusion shown on a distance-neighbour graph. Proc. Roy. Soc. London, 110A, 709-737, https://doi.org/10.1098/rspa.1926.0043.

— J. Meteor., 5, 238-240, https://doi.org/10.1175/1520-0469(1948) $005<0238$ :NOEDIT $>2.0$. CO; 2 .

Rypina, I. I., S. R. Jayne, S. Yoshida, A. M. Macdonald, E. Douglass, and K. Buesseler, 2013: Short-term dispersal of Fukushima-derived radionuclides off Japan: Modeling efforts and model-data intercomparison. Biogeosciences, 10, 49734990, https://doi.org/10.5194/bg-10-4973-2013.

_ - J. K. Llopiz, L. J. Pratt, and M. S. Lozier, 2014: Dispersal pathways of American eel larvae from the Sargasso Sea. Limnol. Oceanogr., 59, 1704-1714, https://doi.org/10.4319/ lo.2014.59.5.1704.

Sarkar, S., H. T. Pham, S. Ramachandran, J. D. Nash, A. Tandon, J. Buckley, A. A. Lotliker, and M. M. Omand, 2016: The interplay between submesoscale instabilities and turbulence in the surface layer of the Bay of Bengal. Oceanography, 29 (2), 146-157, https://doi.org/10.5670/oceanog.2016.47.

Shcherbina, A. Y., E. A. D'Asaro, C. M. Lee, J. M. Klymak, M. J. Molemaker, and J. C. McWilliams, 2013: Statistics of vertical vorticity, divergence, and strain in a developed submesoscale turbulence field. Geophys. Res. Lett., 40, 4706-4711, https:// doi.org/10.1002/grl.50919.

Shroyer, E. L., D. L. Rudnick, T. Farrar, B. Lim, K. Venayagamoorthy, L. C. St. Laurent, A. Garanaik, and J. N. Moum, 2016: Modification of upper-ocean temperature structure by subsurface mixing in the presence of strong salinity stratification. Oceanography, 29 (2), 62-71, https://doi.org/10.5670/ oceanog.2016.39.

Stone, P. H., 1966: Frontogenesis by horizontal wind deformation fields. J. Atmos. Sci., 23, 455-465, https://doi.org/10.1175/15200469(1966)023<0455:FBHWDF>2.0.CO;2.

Sullivan, P. J., 1971: Some data on the distance-neighbour function for relative diffusion. J. Fluid Mech., 47, 601-607, https:// doi.org/10.1017/S0022112071001253.

Thomas, L. N., A. Tandon, and A. Mahadevan, 2008: Submesoscale processes and dynamics. Ocean Modeling in an Eddying Regime, Geophys. Monogr., Vol. 177, Amer. Geophys. Union, 17-38.

Wijesekera, H. W., and Coauthors, 2016: ASIRI: An oceanatmosphere initiative for Bay of Bengal. Bull. Amer. Meteor. Soc., 97, 1859-1884, https://doi.org/10.1175/BAMS-D-14-00197.1.

Zhang, H. M., and Coauthors, 2009: An integrated global observing system for sea surface temperature using satellites and in situ data: Research to operations. Bull. Amer. Meteor. Soc., 90, 8, https:// doi.org/10.1175/2008BAMS2577.1. 